\title{
Study Site Personnel Postal Address
}

National Cancer Institute

\section{Source}

National Cancer Institute. Study Site Personnel Postal Address. NCI Thesaurus. Code C94147.

A contact point used to send physical forms of communication to the study site personnel. 